Meta

Journal des traducteurs

Translators' Journal

\title{
Le néologisme et nous
}

\section{Jean Giraud}

Volume 18, numéro 1-2, mars 1973

Actes du deuxième colloque international de linguistique et de traduction. Montréal, 4-7 octobre 1972

URI : https://id.erudit.org/iderudit/002921ar

DOI : https://doi.org/10.7202/002921ar

Aller au sommaire du numéro

Éditeur(s)

Les Presses de l'Université de Montréal

ISSN

0026-0452 (imprimé)

1492-1421 (numérique)

Découvrir la revue

Citer cet article

Giraud, J. (1973). Le néologisme et nous. Meta, 18(1-2), 225-236.

https://doi.org/10.7202/002921ar

Ce document est protégé par la loi sur le droit d'auteur. L'utilisation des services d'Érudit (y compris la reproduction) est assujettie à sa politique d'utilisation que vous pouvez consulter en ligne.

https://apropos.erudit.org/fr/usagers/politique-dutilisation/
Cet article est diffusé et préservé par Érudit.

Érudit est un consortium interuniversitaire sans but lucratif composé de l'Université de Montréal, l'Université Laval et l'Université du Québec à Montréal. Il a pour mission la promotion et la valorisation de la recherche. https://www.erudit.org/fr/ 


\section{Le néologisme et nous}

L'autarcie linguistique est un mythe : il faut qu'un idiome se frotte aux usages d'autrui, s'instruise en instruisant, domestique des expressions de toutes origines. Perdre ce goût serait chez un peuple le signe de la croissance zéro. Son parler se figerait, captif de son territoire et du passé, comme le deviennent les patois.

Nombre d'auteurs, de voyageurs, de techniciens, de chercheurs ont accueilli ou créé des mots, se sont complu à les manier à l'état neuf. D'autres aussi, écrivains, journalistes, humoristes ont, notamment depuis le milieu du $\mathrm{xrX}^{\mathrm{e}}$ siècle, versé dans la néologie par goût du pittoresque, par maniérisme ou par jeu ; cependant que l'anglomanie littéraire frayait la voie aux emprunts qui, reçus à bras ouverts ou dénoncés trop tard, pénètrent maintenant dans les couches usuelles du langage. Airsi peuvent s'expliquer, grosso modo, la richesse de notre lexique comme ses complaisances.

Les spécialistes de cette branche de la lexicographie moderne furent d'abord des esprits curieux, fureteurs, avancés, tels Louis-Sébastien Mercier (Néologie ou vocabulaire des mots nouveaux, 1801) et Richard de Radonvilliers (Dictionnaire des mots nouveaux, 1845), alors que Littré laissera l'image d'un maître du domaine classique et du purisme ${ }^{1}$.

Deuxième étape : Darmesteter remonte aux lois qui régissent l'apparition des mots ; Maurice Schöne, Georges Gougenheim en parcourent l'histoire, en décrivent la vie, les accidents, la mort; d'André Thérive à Maurice Grevisse, grammairiens et lexicologues engagent dans les revues et dans la presse des dialogues actualisés avec le lecteur; Dauzat fonde le Français moderne; Marcel Galliot cueille sur le vif, avec ses élèves, les bizarreries de la langue de la réclame. Et n'oublions pas, pour l'anglicisme, Fraser Mackenzie, Bonnaffé, Félix de GrandCombe.

Et voici que le troisième âge commence. La chasse aux datations modifie et enrichit le tableau de la néologie historique. Le Français moderne deuxième formule, l'ouvrage collectif sur le Langage dans l'Encyclopédie de la pléiade parlent à l'homme de lui-même, selon la formule de Michel Bréal, cependant que se refondent la terminologie, la méthodologie, l'outillage. Faut-il dire, aujourd'hui,

1. Voir le Robert, t. 4, s.v. néologisme. 
évolution ou drift de la langue ${ }^{2}$ ? Dans quelle mesure peut-on encore parler d'anglicismes au sens étroit? Pour définir le néologisme, suffit-il de distinguer du «terme nouveau » l'acception nouvelle du «terme existant»?

Unité linguistique motivée, susceptible de lexicalisation, le néologisme, aussi humble soit-il, affirme l'exigence créatrice, le besoin qu'éprouve un concept informulé de combler une lacune, dans l'espoir de devenir communicable. Le néologisme d'importation s'ajoute, bien ou mal, au stock historiquement admis par la langue d'arrivée, mais escamote la recherche d'un équivalent : on a dit de l'emprunt qu'il nie la traduction, du calque qu'il camoufle une impuissance ; tel est le jeu de la loi du moindre effort. De toute façon il doit — ou devrait perdre son opacité initiale en devenant transmissible.

Enfin, selon que nous ouvrons les portes toutes grandes ou les verrouillons, que nous lâchons les chiens fous ou les tenons en laisse et muselés, nous donnons, vis-à-vis du nouveau venu, soit dans le laxisme qui autorise tout en ne régularisant rien, soit dans le rigorisme. René Doumic écrivait sereinement en 1915 : "Il appartient à l'Académie française de s'opposer à l'écoulement de la langue.» Autrement dit, le dilemme serait d'accélérer ou de freiner le cours d'un fleuve dont le débit, depuis lors, s'est accru démesurément.

Au lieu de nous gratter la tête en murmurant : «Diable, diable... », essayons un peu d'y voir clair.

Tout néologisme naît à ses risques et périls : «Sera-t-il dieu, table ou cuvette ? $\gg$ Ses espérances de vie sont capricieuses, mouvantes, sujettes à éclipses, à naufrages, à résurgences. Ainsi, j'ai rouvert dernièrement un fichier établi il y a une dizaine d'années au hasard de mes lectures, et devant lequel je me demandais alors : "Où vas-tu, français contemporain ? "Or, déjà les déchets y foisonnent, victimes des batailles ou des gaspillages de mots. Certains de ceux-ci ont glissé à l'arrière-plan et seront rarement repris. D'autres étaient des stropiats de naissance. Et plusieurs n'ont pas eu ce que les sportifs appellent la baraka ou protection divine, la surabondance verbale ayant pour rançon des chutes parfois imméritées. Jusqu'à quel point nous en affligerons-nous? La sélection naturelle limite, par une sorte de sagesse latente, les excès et les dégâts de la surproduction. Comme dit la chansonnette :

Elles font, font, font,

Les petites marionnettes,

Elles font, font, font,

Trois p'tits tours, et puis s'en vont...

Je ne m'étendrai donc pas sur le logos irrévérent, abstrus, étiré, tronqué, biscornu, canularesque, ou ramené à l'orthographe phonétique, qu'atteste ce fichier drôle et exacerbant, florilège, au demeurant trop peu représentatif de la langue d'usage pour qu'il atteigne le seuil de la contamination.

2. L'intensif drift marque à la fois l'idée de pulsion, d'entraînement, de charriage et celle de perte de contrôle : le langage part à la dérive; on ne sait à quoi son mouvement aboutira. 
Permettez que j'estime plus significative la chasse aux Mots dans le vent. Il ne s'agit plus d'un simple regard promené sur l'étrangeté et le snobisme, mais d'un petit dictionnaire de pointe de la néologie la plus récente : «Un instantané sur le mouvement du vocabulaire $»$, m'écrivait mon très regretté maître et ami Georges Gougenheim. Il fait prendre date, soit à des vocables en début de carrière, donc sujets aux périls que je viens d'évoquer, soit à une polysémie galopante. Il essaie d'éclairer quiconque, chaque jour, lit ou entend des formes opaques contre lesquelles il bute. Certaines passeront comme le café ; d'autres se fortifieront, feront souche, changeront de sens, que sais-je? Ne cherchons pas trop à préjuger l'avenir. Ce recueil, donc, est un poste de décryptage, de dépannage ou, comme dit Pierre Pamart, de simple mise en observation. Ajouterai-je que nous n'avions pas à condamner formellement, fût-ce pour des raisons évidentes, ces produits « de la mode et de l'actualité »? Un dictionnaire enregistre, clarifie, informe, mais ne légifère pas. La critique doit apparaître en filigrane et, de préférence, par le biais discret de l'humour.

Précisons notre propos. Tâchons de voir, à l'aide d'un choix très insuffisant de remarques et d'exemples, comment s'habille, se présente, se comporte le néologisme contemporain. Quel auteur n'a jamais éprouvé le besoin d'en fabriquer, d'en saisir un par l'oreille? Qu'ils sont passionnants ces alpages, ces forêts, ces grands fleuves de mots qui narguent, déconcertent l'observateur, jouent à cachecache avec lui tels des papillons, s'enveloppent de manteaux ou de livrées étranges, ou bien fondent sur nous pêle-mêle! Comparons :

Le français incline, semble-t-il, plus que l'anglais, à néologiser en raison de son système grammatical moins flexible. Lorsque le composé science-fiction précède un substantif anglais il a valeur de déterminant, de qualifiant. Cette possibilité nous manque : alors, nous tombons en arrêt devant les prouesses de l'épopée science-fictionnesque. Un néologisme de plus : d'autres suivront.

L'anglo-américain n'a sans doute ni traqué ni proscrit ses polysyllabes puisque, il y a quelque cinquante ans, les auteurs du King's English les malmenèrent sans résultat notable. Toutefois, il concilie souvent le laconisme et la clarté : songez au théâtre off ou off-off, à be-in, bed-in, sit-in, teach-in, à in tout court, à must substantivé, à $i t$. Le français, quant à lui, chemine encore trop volontiers dans la foulée du fameux phénakistiscope (1833) du Wallon Josëph Plateau, maintes fois déformé ensuite comme risquent de l'être les mots imprononçables. C'est pourquoi l'on trouvera dans la prochaine cuvée des Mots dans le vent : contraventionnalisation, conversationnel, nationalisationite, pédobaptisme, après-tsirananaïsme et autres pythons déroulant avec joie leurs anneaux au soleil. Cette morphologie étirée est cependant battue en brèche par la riposte juvénile des maxi, des midi, des mini, des majo, des mino, des mao, des réviso et autres apocopes : il faut de tout pour faire un monde...

Les modes et les vogues de nos affixes apportent force indications sur les distinguos $\mathrm{du}$ vocabulaire. Considérons les préfixes anti, contre et $-d e$, très actifs dans la formulation contestataire : 
anticulture, sens négatif plein; antipollution, antipsychiatrie, réaction positive; antivacances, sens négatif atténué (les vacances prises après le retour des autres) ;

contretout, nihilisme; contre-culture, contestation limitée à la culture de grand-papa ; contre-information, critique positive (information parallèle susceptible de compléter ou de corriger) ;

désétatisation, désescalade, renversement de la vapeur; décélération, simple ralentissement forcé, et que l'on espère provisoire, d'une progression; désacralisation, ce mot a déjà tellement servi qu'il s'émousse de jour en jour.

Ainsi nous ne pouvons attribuer ni les mêmes fins, ni des valeurs identiques, à tous les emplois d'un préfixe.

On trouve aussi quelque désordre dans la jungle suffixale. Si compétitionnite, électoralite, téléphonite, maladies ou servitudes contagieuses de notre temps, sont très clairs, on peut s'interroger devant sinistrologue ou victimologie; et quelle commune mesure existe-t-il entre la médiathèque publique, ou la joyeuse ludothèque au service de l'enfant, et l'érothèque, commerce prétentieusement camouflé ?

Autre phénomène : le mimétisme par attraction paronymique : négritude $\rightarrow$ FEMELITUDE; inflation $\rightarrow$ STAGFLATION; explosion $\rightarrow$ SEXPLOSION; funambule $\rightarrow$ LUNAMBULE ; vespasienne $\rightarrow$ VESPACHIENNE.

Je passe et arrive à la plus importante, sans doute, de ces constatations. Il s'agit du néologisme notionnel, ainsi appelé parce qu'il ne concerne pas un mot, mais l'expression d'une idée nouvelle à travers une association inédite de termes. On le rencontre surtout dans la presse, dans l'exposé des thèses politiques ou sociologiques, et dans plusieurs titres d'ouvrages. Il dramatise plus souvent qu'il ne coule en euphémismes les inquiétudes, les mises en condition, les illogismes, les images spectaculaires ou émotives de notre époque, comme le montre ce relevé très partiel :

bain de foule - de peuple
changer la vie (une « résurgence » d'Arthur
Rimbaud)
la créativité encagée
la démocratie supplétive
l'érosion du plein emploi 3
l'exode des cerveaux
la fuite en avant
la gauche du Christ
les grossistes des vacances
l'ivresse chimique
le journal téléguidé
le marketing des urnes
la mort de Dieu

la nation sur roues un peuple programmé la pollution génétique, psychique, sonore, de l'argent... récupérer les irrécupérables les silos à vivres socialiser la nature la société bloquée, câblée, de rendement... la sociologie des gouffres le théâtre éclaté le tiers monde des mots l'univers pavillonnaire la vieillesse sociale...

Bon ou mauvais, le néologisme est par conséquent révélateur sous toutes ses formes. Il fait de la néologie un domaine non plus seulement morphologique et descriptif, mais une science à l'écoute de l'univers, auxiliaire de la recherche

3. C'est-à-dire le chômage. 
comme de la réflexion. N'est-ce pas là une des grandes orientations que ne saurait ignorer notre Colloque?

J'aborde maintenant le néologisme d'importation - avec précaution et modestie, car vous êtes, au Québec, orfèvres en la matière ainsi que le montre en particulier votre journal des traducteurs : META. Ces questions vous sont tellement plus familières qu'à nous !

Rappelons d'abord que l'on accuse assez légèrement le français d'être la plus emprunteuse, la plus pilleuse des langues de grande communication. En effet, l'anglais du $\mathrm{XIX}^{e}$ et $\mathrm{du} \mathrm{Xx}^{\mathrm{e}}$ siècle abonde en gallicismes; et dans un récent Dictionnaire allemand des mots étrangers on relève 15000 éléments de base et le clouble de dérivés, dont les deux tiers sont français. Le mouvement n'est donc pas à sens unique.

Faisons la part du feu. Nul ne songe à proscrire, comme le voulait il y a un siècle l'académicien Viennet, club, redingote, tunnel ou wagon. L'emprunt est inévitable. Certains le justifient, au moins dans sa fraîcheur, par une sorte d'aura, de tonalité, de couleur locale propres à nous transporter psychiquement ailleurs, à nous dépayser. Il porte quelquefois aussi des valeurs auxquelles on trouve malaisément des correspondances exactes : establishment, gap, happening, lipservice, permissive society, understatement, video-party. À charge de réciprocité, car nous sommes munis en gallicismes adoptables. Mais l'essentiel ne tient évidemment pas dans ce petit import-export.

«La France, constatait Pierre-Henri Simon en 1962, n'est plus la seule responsable de sa langue et de sa culture. » $Y$ serions-nous consentant? Il n'est que de lire ce bout de réclame : "La technique New hair, découverte par des spécialistes parisiens... »Si des spécialistes parisiens préfèrent New hair à « cheveux tout neufs », alors, laissons-nous saturer de fausses élégances et d'exotisme à quatre sous. Mais disons-nous que c'est par soumission à l'impact de facteurs extra-linguistiques que le français devient langue subalterne.

«La vie exige, affirme Aurélien Sauvageot, que la langue transmette le maximum de quantité d'information dans le temps le plus court et dans les conditions les plus sûres. » Techniquement les plus sûres, soit. Mais elle nous parvient à l'état barbare, polluée et polluante, grouillis de surenchères verbales qui se télescopent sous la boîte crânienne de l's Hexagonal » moyen, incapable de s'y reconnaître. On ne lit plus ; on regarde, on entend, privé que l'on est des pauses, des parenthèses d'assimilation que ménage une saine lecture. Un mot insolite chassant l'autre, il faut ou bien comprendre sur le champ, ou bien renoncer à comprendre : c'est la négation du dictionnaire. Il manque une ligne directrice de rééducation linguistique et jusqu'au garde-fou de l'« aide-toi, le ciel t'aidera ». L'assainissement du langage demande une politique, un gentlemen's agreement visant à contingenter - je ne vois pas d'autre terme - l'entrée des mots étrangers qui ont le courage de se présenter à découvert.

Quant aux autres, aux anglicismes honteux où Marcel Galliot voit le plus grave danger : agressif, approche, auditeur interne, contrôle des naissances, 
ensemble avec, les coloris explosifs, le peeling (ah! ces noms verbaux), les procédures industrielles, les ventes promotionnelles, la réhabilitation au travail, les hivers sévères et tant d'expressions de même farine, nous ne redirons jamais assez qu'elles sont superfétatoires - et lâches, puisqu'elles prennent sans défense le locuteur non angliciste.

D'autres cas aussi encourent le refus, mais pour des raisons toutes différentes :

D'abord, évidemment, lorsque la traduction devrait aller de soi : suite, appartement; house-organ, journal interne, « journal-maison ». Mais les faits démentent la raison : libre-service, donné par les dictionnaires d'usage et ratifié par 93\% des suffrages à la Biennale de Menton, commence seulement à grignoter selfservice. Armons-nous donc de patience ;

Lorsque, à l'opposé, le mot de départ présente une construction trop caractéristique pour se prêter au calquage : breakfast news, cash and carry, stop and go driving. Il faut transposer - en moins concret ;

Quand le remplaçant, même imparfait, demeure préférable au mot de départ opaque : brain-drain, exode ou fuite (littéralement, pompage) des cerveaux; jointventure, entreprise à disques conjoints. Le Suisse Jean Muratet va jusqu'à souhaiter que «la préférence soit chaque fois donnée au terme français », celui-ci serait-il «moins courant et moins bien compris que le terme anglo-saxon 》 (Vie et langage, $\mathrm{n}^{\circ}$ d'août 1972, p. 479) ;

D'autre part, le vieux calque morphologique permet des accommodements : actuariel, footballeur, interviouveur. Il en va de même du calque sémantique plus subtil. Louis Arman'd n'avait pas si mal trouvé son remue-méninges pour brainstorming, à peu près inassimilable en français. Cet équivalent un peu facétieux, ce qui facilite l'adoption, a été repris récemment dans notre quotidien le mieux écrit : le Monde.

Deux possibilités sont encore offertes :

La résurgence, dans la polysémie actuelle, de vieux mots refaisant surface après un long oubli ou demi-oubli : farfelu, fiabilité/fiable, maintenance, pollution, usance;

Et la logoplasie ou confection, façonnage de «paroles». Lorsque, notamment dans le domaine scientifique, technique ou professionnel - où la création consciente a toujours tenu un rôle de premier plan ${ }^{4}$ - le terme de départ demeure opaque et si les équivalents déjà proposés ne satisfont guère - à plus forte raison s'il s'agit d'une notion toute nouvelle - alors il faut qu'un groupe de travail s'attaque de concert à la difficulté. C'est un problème que vous connaissez bien puisque la terminologie de langue anglaise vous enveloppe (je songe notamment aux fameux tandems hardware-software et marketing-merchandising). Confectionner le mot qui offre le plus d'espérances de vie parce qu'il sent moins que d'autres l'embarras ou le préfabriqué, c'est éviter, en les devançant, les malfaçons et les échecs. La

4. Voir, par exemple, notre «Contribution à une étude de la langue de la télévision en français », référencée en bibliographie. 
création à plusieurs devient l'une des tâches les plus absorbantes de votre École de traduction comme du Comité d'étude des termes techniques français.

Loin de moi la croyance naïve aux panacées. Je me garderai de dire avec J. O. Grandjouan qu' une langue est toujours victorieuse de ses emprunts »; mais il me semble que le bon emploi de la traduction et de la francisation d'une part, celui de l'équivalence et du remplacement d'autre part, celui enfin de la logoplasie, permettent de déblayer en grande partie le chemin. Vouloir, c'est pouvoir. Si nous ne court-circuitons pas l'indolence, si nous ne secouons pas le laisser-aller, si nous n'offrons pas des solutions que nous aurons cherchées nousmêmes, alors nous progresserons à tout petits pas. L'opération des « vingt termes les plus contestés de la langue des affaires », conduite à la Biennale de Menton en septembre 1971, a montré qu'il est possible "de mettre fin à des discussions byzantines » et de s'entendre «sur des substituts acceptables», comme l'affirmait avec sa robuste franchise notre cher Alain Guillermou. Que nous fassions individuellement des réserves sur tel ou tel des équivalents proposés, c'est inévitable. L'essentiel est d'avoir prouvé que l'anglicisme n'est pas sacro-saint.

J'en arrive à mon deuxième point.

Amis québécois, j'ai souvent médité sur votre situation linguistique, sur vos efforts et sur les nôtres, en me demandant comment les harmoniser. J'ai salué la mémoire du devancier anonyme, sans doute ouvrier typographe, qui, en 1860 déjà, publiait à Québec son Recueil des expressions vicieuses et des anglicismes les plus fréquents.

Nul ne l'ignore, le contexte bilingue et biculturel dû à votre position géographique au poste le plus avancé de la francophonie vous a, de longue date, sensibilisés. Parlons en toute objectivité. Vos lexicographes, frères de ceux dont j'évoquais les noms tout à l'heure, ont compris qu'il ne s'agit plus uniquement de protéger le fond français, précieux symbole de votre patrimoine et de vos traditions. Vous êtes en quelque sorte surexposés, dans vos réclames, dans le parler quotidien de la serveuse de restaurant, du mécanicien, du sportif, dans toutes vos relations de vocabulaire, traductions comprises, au recouvrement de votre idiome vernaculaire par votre langue seconde, belle et grande elle aussi, mais profondément autre. Et ce processus tend, d'une part, à substituer les structures lexicales et syntaxiques du pays voisin à celles du français et à faire de celui-ci un anglo-américain déguisé ; d'autre part, à imposer un régime anglophone du néologisme, notamment dans les terminologies de métiers.

À cet état de fait vous réagissez avec force. Au «français » aberrant de tel journal que je ne veux pas nommer vous opposez le français irréprochable de Forces. Vous mobilisez l'immense appareil de vos télécommunications, faites donner votre Office de la langue française, vos Études françaises, votre Académie canadienne-française, vos presses universitaires. Sachant que prêcher des convertis c'est piétiner, vous avez constitué des équipes, recensé vos canadianismes de bon aloi, édité des fichiers usuels ou techniques hautement appréciés chez nous, des lexiques, des glossaires professionnels, lancé avec Robert Dubuc l'opération Objectif 200 , bref, alertement cimenté toute la base de l'édifice, au niveau où le gallicisme même ancien et éprouvé redevient en fait, pour l'homme démuni 
de la rue, un néologisme ignoré, révélé, une redécouverte du parler franc et droit. Et j'associerai dans un même hommage vos publications technico-scientifiques, tels le Lexique de gestion ou le Dictionnaire bilingue de l'informatique, et l'œuvre accomplie par votre École des traducteurs et votre Banque de terminologie, avec ce fichier dont l'interrogation et la démonstration couronneront cet après-midi notre Colloque. De votre esprit d'initiative, de votre sens de l'efficacité, soyez complimentés en langage sans fard. Toute notre affection soutient et encourage votre effort.

Quant au «vieux pays», il est joyeux et fier de mettre à votre service la gamme de ces ouvrages dont Jacques Cellard écrivait récemment qu'ils renouvellent les études de lexicologie en attestant «l'effervescence heureuse de notre vocabulaire national $\gg$. Il s'agit, bien entendu, du nouvel âge des dictionnaires dont Paris est l'âme et l'usine. Des titres par centaines! Jusqu'à un Dictionnaire des injures en édition de luxe : noblesse oblige... Restons sérieux. Pour nous en tenir au domaine proprement linguistique je dénombre, sauf omission, chez Larousse seulement : onze titres en un volume, trois en plusieurs et vingt-deux spéciaux.

Le premier dictionnaire est celui que nous n'avons pas à ouvrir puisque nous le portons en nous : notre idiolecte ou lexique personnel, extrêmement variable, suivant l'âge et le milieu, en pourcentages de néologismes et d'éléments exogènes. Un idiolecte de 25000 termes est exceptionnel, donc enviable : c'est le triple environ du vocabulaire fondamental de la communication sans les langages techniques. Cependant il le cède de loin, par exemple, aux quelque cent cinquante mille articles du Dictionnaire français de médecine et de biologie que vient de publier, avec le concours de 350 confrères, le docteur roumain Manuila. Mais il s'agit là d'un recueil de consultation et de référence, beaucoup plus éloigné du vocabulaire général que ne l'est le premier tome de l'Essai d'orientation de la terminologie élaboré par le Comité d'étude des termes techniques français, à l'intention de nombreux corps de métiers. Tournons-nous donc vers les ouvrages non spécialisés.

Un dictionnaire d'usage tel que toute famille devrait en posséder demande au moins 40000 entrées et s'augmente d'une révision à l'autre. Après ce minimum, l'escalade irréversible commence. Pour simplifier, comparons les trois recueils géants de la linguistique actuelle, édités en tout ou en partie : le Grand Larousse de la langue française, qui s'adresse «aux francophones cultivés, du niveau élémentaire au niveau supérieur, et de tous les niveaux sociaux », atteindra 70000 entrées; le Trésor de la langue française, qui explore surtout la langue littéraire du XIX et du $\mathrm{XX}^{\mathrm{e}}$ siècle, en présentera 80000 ; le gros Robert, fruit de vingt années de labeur, en atteignait 120000 avant l'édition de son Supplément, où l'on trouve en particulier des milliers de néologismes.

Devant cette montée des chiffres, devant les millions d'exemples recensés électroniquement, on comprend pourquoi le Littré, monument et prouesse d'un autre âge, est dépassé ; pourquoi aussi l'on pourrait appeler le patient travail de nos académiciens le plus mignon de nos dictionnaires de prestige... 
En présence de ces masses de mots une constatation me paraît s'imposer. Si le plus complet des dictionnaires aura toujours ses lacunes, celles-ci concerneront désormais presque exclusivement le passé, j’entends les écrits maintenant perdus que les méthodes et les moyens d'autrefois n'ont pu atteindre. Par conséquent et c'est là que je voulais en venir - le travail des équipes de dictionnaires deviendra de plus en plus celui de néologistes mettant en ordre et en forme les mots naissants, le langage tel qu'il vient de se créer. Et les besoins ne tariront jamais.

Ainsi, Québécois ou Français de France, nous pouvons nous donner l'accolade : nous avons fait du bon ouvrage en lexicographie. Grâce aux outillages qui multiplient les perspectives de recherches, le néologiste devient l'homme de pointe d'une maîtrise totale, ou presque, du langage — ce qui ne s'était jamais vu.

Essayons de conclure.

1) Les recueils de la langue d'actualité, de la langue «incipiente » comme dit fort bien l'anglais, permettent - malgré les critères de validité encore incertains - de mieux filtrer les passages des éléments les plus stables de la multitude néologique vers les dictionnaires à grande tomaison comme vers les dictionnaires d'usage.

2) Témoin du mouvement du vocabulaire, le chasseur-dépouilleur de néologismes et d'emprunts est une sorte de trait d'union entre le dépistage et l'analyse du mot, et la surveillance de sa circulation et de son destin.

3) Ne doit-il pas prendre langue, soit pour informer, soit pour s'informer, avec tous ceux qui peuvent ajouter le courant de la vie au dépouillement des textes : le journaliste, le publicitaire, l'animateur de club de jeunes, le responsable d'œuvres socio-éducatives, de cours de chambre de commerce, l'instituteur, le professeur de collège technique dont les élèves baignent dans un milieu de langage pollué ?

4) Établirons-nous bientôt, à l'intention de ceux pour qui l'assainissement du langage est un moyen d'《apprendre à être», notre propre Objectif 200 à partir des fréquences d'usage et des gravités prioritaires, ne serait-ce d'abord qu'à titre expérimental?

5) Il ne m'est pas possible de développer ici la question vers laquelle converge finalement tout mon propos ${ }^{5}$ : celle de l'enseignement - au sens large - de la langue (je ne dis pas de la littérature) et notamment du français contemporain, avec son préalable : y préparer, $y$ former les enseignants.

Il me suffira de constater, avec beaucoup d'autres, ce paradoxe : au moment où l'acquisition des langues étrangères et celle du français langue étrangère commencent à passer, chez nous, par des méthodes et des outillages efficaces, l'orientation du plus grand nombre possible de nos compatriotes vers une meilleure

5. Cf. J. Giraud : "La norme, l'usage et l'enseignement du français », Vie et langage, nos 231 et 232 , juin et juillet 1971 . 
pratique de leur idiome dans les relations courantes de la vie, demeure presque ignorée. La lecture, l'audition, l'écriture, la langue des spectacles et des jeux, celle des affaires, sont saturées de néologismes; la langue scientifique rend au latin et au grec une manière de seconde vie par de nouvelles utilisations des racines et des affixes ; l'anglicisme pavoise les vitrines du pharmacien, du coiffeur, du marchand de journaux; les adolescents et même les enfants néologisent entre eux : et nous attendons encore, du moins à ma connaissance, l'ouvrage limpide, pratique, révélateur qui entreprendrait d'éduquer nos façons de nous comporter vis-à-vis de ce grand phénomène social qu'est l'accélération du langage ; le livre bien fait pour têtes bien faites qui rassurera son lecteur au lieu de l'effaroucher.

Dans les Hommes et la technique (1931), l'Allemand Osvald Spengler distingue deux étapes dans l'histoire des techniques. En éduquant sa main l'homme a d'abord conquis son environnement, mais par ses seules ressources, comme l'eût fait un animal. La main supposant l'outil, il est passé au stade de l'outil sans se libérer encore de l'individualisme, du combat précaire pour subsister. Vint le pouvoir de se manifester à autrui : la civilisation du langage. Peu importe à Spengler comment celui-ci s'est constitué. L'essentiel, c'est qu'il ait fait de l'individu un être social, capable de comprendre et d'être compris, un être de sentiments, de jugements de valeur, d'actions concertées. La lutte contre la nature a dès lors revêtu une ampleur grandiose.

C'est parce que nous ne pouvons rien sans le langage que ce dernier, continûment, s'est enrichi et socialisé en tant que moteur de l'imagination agissante. Mais, plus il se diversifie dans sa spécialisation croissante, plus il s'expose aux accidents que nous voudrions prévenir. La coexistence des communautés linguistiques d'aujourd'hui avec leur afflux de mots de travail, de loisir, de culture, de science, provoque en effet des surplus quand ce ne sont pas de fausses élégances, des trompe-l'œil ou des attrape-nigauds. Nous sommes les vérificateurs de cette circulation dont les dictionnaires sont les vastes charrois. Nous sommes les douaniers vigilants mais discriminatifs de l'entrée du mot sur nos territoires. À nous d'empêcher que l'énorme mouvement du vocabulaire obscurcisse - que dis-je ? souille de sa marée noire, et la fiabilité de chaque élément de l'outillage, et la personnalité francophone comme domaine d'expression.

Le mot a toujours eu partie liée avec nos civilisations et leurs conquêtes. Que ses malfaçons, ses redondances, ses débordements d'aires sémantiques, ses abus de pouvoir ne nous fassent jamais perdre de vue la vérité antique et fondamentale de l'outil, serviteur du couple cerveau-main. Ne le laissons jamais se dévitaliser, s'abâtardir, s'enlaidir dans la confusion et le bavardage, au moment où l'essor linguistique commence à lui dresser des monuments qui rassembleront tout le passé, tout le présent, tout le futur des activités de notre espèce. Mais gardons-nous aussi d'entraver à l'excès son développement naturel. $\mathrm{Si}$, comme le soleil, le beau et franc langage brille pour tous, s'il est la condition d'un humanisme, alors, discipliner avant qu'il soit trop tard le néologisme d'aujourd'hui, envahisseur trop zélé, c'est préparer à nos petits-enfants le champ lexical sans 
ivraie dont ils auront besoin à leur tour. Faisons nôtre la devise de la ville de Paris : Fluctuat nec mergitur. Sans doute le vaisseau est-il battu par les vents, mais il ne sombrera pas.

JEAN GIRAUD

\section{BIBLIOGRAPHIE RÉCENTE}

\section{Génêrale}

ACHARD, P., «La linguistique, pourquoi ? ", l'Education, publication du ministère de l'Education nationale, Paris, 14 mai 1970

Cel.tard, J., « Français, franglais, Europe », le Monde, 29 décembre 1971.

CeldARD, J., "Anglicisme et logologie », le Monde, 2-3 juillet 1972.

EYRAUD, Dr D., "Vive la langue ! ", Vie et langage, avril 1969.

GerouvrIER, E., «Que doit-on, que peut-on enseigner? ", le Monde, 10 septembre 1969.

Giraud, J., "Contribution à une étude de la langue de la télévision en français ", Cahiers d'études de radio-télévision, juin 1960, p. 162-191.

GIRAUD, J., « Réflexions sur les anglicismes techniques », Vie et langage, avril 1969.

Gougenherm, G., les Mots français dans l'histoire et dans la vie, 2 vol., Paris, A. et J. Picard, 1966 et 1968.

Gutlbert, L. (dirigé par), « Le lexique », Langue française, mai 1969.

PAMAart, P., * Néologismes », Vie et langage, juillet 1971.

S.N.D.A., "Les mots qui courent", néologismes et anglicismes dans le vocabulaire de la mode, Vie et langage, août 1971.

SAUVAgeot, A., Portrait du vocabulaire français, Paris, Larousse, 1964.

Dictionnaires spéciaux

Collectif, Termes techniques, essai d'orientation de la terminologie, Comité d'étude des termes techniques français, Paris, Hartmann, 1972.

Ducrot, O. et T. TODorov, Dictionnaire encyclopédique des sciences du langage, Paris, Seuil, 1972.

GILbert, P., Dictionnaire des mots nouveaux, Paris, Hachette-Tchou, 1971.

GiraUd, J., P. Pamart et J. Riverain, les Mots dans le vent, Paris, Larousse, 1971.

Sur les dictionnaires

Cellard, J., «Qu'est-ce qu'un dictionnaire ? ", le Monde, 30-31 janvier 1972.

Cellard, J., « Un dictionnaire nouveau », le Monde, 4-5 juin 1972.

Dubois, J., L. Guilbert, H. MitTerand et J. Pignon, "Le mouvement général du vocabulaire français de 1949 à 1960 d'après un dictionnaire d'usage ", le Français moderne, avril 1960 .

* Entretien : comment Paul Robert a imaginé et réalisé son dictionnaire. Propos recueillis par Paul Morelle », le Monde, 10 mai 1967.

GUILLERMou, A., " Médecine et langage ", Vie et langage, avril 1972.

Le EitboIs, R., «A propos d'un nouveau Larousse », le Monde, 21-22 novembre 1971.

Matoré, G., Histoire des dictionnaires français, Paris, Larousse, 1971. 
"Que trouve-t-on en faisant l'inventaire de la langue française? Une interview de Forces avec Paul Robert », Forces, n' 17, 1971.

ReY-Debove, J., Etude linguistique et sémiotique des dictionnaires français contemporains, La Haye-Paris, Mouton, "Approaches to Semiotics», 1971.

Sur le Trésor de la langue française

CellaRd, J., "Le Trésor de la langue française : le premier tome d'un grand inventaire", le Monde, 18 février 1972.

Le Français moderne, depuis le $\mathrm{n}^{\circ}$ d'avril 1968 : Textes traités par l'ordinateur. - Les structures lexicales empiriques du francais. - La mécanisation de la documentation bibliographique. - Le bulletin analytique de linguistique française. - Inventaire général de la langue française. - Méthodologie générale du T.L.F. - Le traitement des éléments grammaticaux et des éléments formateurs..

\section{QUESTIONS}

N... : N'avez-vous pas l'impression que, dans certains cas du moins, plus la formation et l'utilité d'un néologisme sont évidentes moins ce néologisme a de chances d'acquérir rapidement droit de cité ? Quels critères précis adoptez-vous pour enregistrer tel ou tel néologisme?

Réponse : Il se peut fort que vous ayez raison dans de nombreux cas, mais il y a des exceptions. Les mots "reprographier", "reprographieur ", "reprographique " sont, à mon avis, des termes excellents. "Reprographier " est plus précis que " duplicater ». Nous aurons peut-être "reprographiste ". Cette chaîne de dérivés montre que le mot a vraiment une utilité, une fonction et il me semble qu'il a progressé très vite dans l'usage.

M. Giraud : "Les mots dans le vent ", c'est une sorte de pré-dictionnaire, d'avant-dictionnaire. Il faut tenir compte surtout des fréquences d'emploi et évidemment du pifomètre, dans une certaine mesure. Il n'est pas question d'employer les moyens scientifiques pour établir des ordres de fréquence. Nous n'avons pas à proposer des mots à tel ou tel groupe professionnel; nous pouvons en recommander certains. Parmi les néologismes, il y en a qu'il faut tout de même suivre avec intérêt et dont il faut essayer de favoriser le passage. Il y en a d'autres, qui sont de simples curiosités ou qui sont des mots mal faits, par exemple «médiocratie, gouvernement par les médiocres ". Il suffit de se reporter à l'étymologie, la \& médiocratie" se serait le gouvernement par les gens des classes moyennes et non le gouvernement des médiocres. 